\title{
A study to assess the use of fibre-optic transillumination in the general dental practice
}

\author{
The use of fibre-optic transillumination in general dental practice by G. M. Davies, H. V. Worthington, J. E. Clarkson, \\ P. Thomas, R. M. Davies Br Dent J2001; 191: 145-147
}

\section{Objective}

To assess the use of fibre-optic transillumination (FOTI) as a diagnostic tool in general dental practice.

\section{Design \\ A cross-over quantitative study for the diagnosis of approximal carious lesions by two clinical methods with a qualitative component.}

\section{Subjects and methods \\ Seven GDPs were trained to use FOTI as an adjunct to their usual clinical examination to diagnose approximal caries. After 12 weeks of use in their practices four of the GDPs took part in two assessment sessions, set a week apart, using 29 volunteer patients. Each patient was examined on two separate occasions by each GDP using either their standard clinical examination technique alone or supplemented by FOTI examination. The order of the techniques was randomised. Radiographs of each patient were also examined separately. An experienced FOTI user also examined the patients to provide a benchmark. From standard charts the number of enamel and dentinal lesions on approximal surfaces was summed and comparisons made between the techniques. Six GDPs took part in one-to-one interviews.}

\section{Main outcome measures}

Mean numbers of carious lesions recorded by each clinician using each technique.

\begin{abstract}
Results
There was a trend for all GDPs to find more enamel and dentinal lesions using FOTI, than with their standard clinical examination with or without radiographs. All GDPs found the FOTI technique a useful adjunct. They used FOTI in different ways and found a variety of uses for it other than caries diagnosis.
\end{abstract}

\section{Conclusions}

The FOTI technique increased the detection of approximal carious lesions. It was reported to be a useful diagnostic tool in general dental practice. GDPs and staff enjoyed participating in a research project.

\section{In Brief}

- GDPs can use FOTI to enhance diagnosis of approximal lesions if training is given and the technique is practised.

- GDPs found a variety of clinical uses for FOTI, apart from caries diagnosis.

- When academic researchers are planning research with GDPs the demands of the two different working environments must be taken into consideration to achieve a successful and meaningful outcome.

\begin{abstract}
Comment
$\mathrm{C}^{\text {ibre-optic Transillumination (FOTI) as }}$ a diagnostic tool is not new. The objective of this study, however, was to evaluate the tool in the primary care setting and to gain some feedback from general dental practitioners regarding the value of the method on a day-to-day basis.

A small sample of 4 dentists participated in the main part of the study in which they all assessed interproximal sites in 29 volunteers on 2 separate occasions; clinical examination alone was used at one visit and this was supplemented with FOTI on the other occasion. Blinded examinations of recently taken bitewing radiographs were also examined. When the standard clinical examination was supplemented with FOTI, one of the dentists found significantly more approximal enamel lesions compared with using the clinical examination alone. With respect to dentinal lesions, 2 general dental
\end{abstract}

practitioners found significantly more lesions when FOTI was used with the clinical examination. When the clinical examination was undertaken in conjunction with radiographic examination, the detection of both enamel and dentine lesions increased. Again, supplemental FOTI increased significantly the number of enamel lesions detected by one dentist and the number of dentine lesions by 2 practitioners.

Feedback from the general practitioners suggested that FOTI is a useful tool for detection of approximal caries although the device used in the trial was apparently quite bulky and cumbersome, and would need modification to be more 'streamlined' to the dental surgery environment.

Although this is a relatively small study, it demonstrates very well how potentially valuable clinical diagnostic tools can be evaluated in the primary care setting. It is also important to note from the data that adjunctive use of FOTI is unlikely to lead to a significant increase in placement of restorations. It might provide the clinician with the opportunity of diagnosing previously undetected, incipient interproximal lesions that can be managed early by a preventive approach.

Perhaps as a further consideration, it would be interesting to know the sensitivity and specificity of FOTI as a stand-alone diagnostic technique in primary care; falsepositive and false-negative observations have clinically significant implications both to patients and clinicians. This information will, I am sure, become available in due course.

\section{Dr P. A. Heasman}

Dental School

University of Newcastle upon Tyne 